\title{
DOMAIN FORMATION OF TbFeCo FILMS ON A SMOOTHED UNDERLAYER SURFACE
}

\author{
J. Ushiyama, H. Awano' ${ }^{1}$ H. Miyamoto, K. Andoh, H. Sukeda and M. Takahashi \\ Central Research Laboratory, Hitachi, Ltd. \\ 1-280 Higashi-Koigakubo, Kokubunji-shi, Tokyo 185, Japan \\ ${ }^{1}$ Information Media Research Laboratory. Hitachi Maxell, Ltd. \\ 6-20-1 Kinunodai, Yawara-mura, Tsukuba-gun. Ibaraki 300-24, Japan
}

\begin{abstract}
To obtain a high S/N magneto-optical disk with a flat underlayer, the motion of the domain wall and the recording noise on various $\mathrm{TbFeCo}$ disks were investigated while varying compensation temperature and underlayer surface roughness. In disks with a rough underlayer, the recording noise decreased with increasing compensation temperature. In disks with a flat underlayer, we found that a rare-earth-rich $\mathrm{TbFeCo}$ disk had a large recording noise because of a strong contracting force acting on a domain wall. The relationship between the TbFeCo composition, the domain size and the contracting and expanding forces acting on the domain wall in the disks with a flat underlayer can be explained using Huth's theory. In the disks with a rough underlayer, however, the dependence of these forces on composition and domain size could not be explained using Huth's theory. A possible cause of this difference in the domain wall motions of the disks with flat and rough underlayers is that a disk with a flat underlayer has few domain wall pinning sites than a disk with a rough underlayer.
\end{abstract}

KEYWORDS: MAGNETO-OPTICAL DISK, RECORDING NOISE, UNDERLAYER SURFACE ROUGHNESS, CONTRACTING FORCE, EXPANDING FORCE, DOMAIN WALL PINNING SITE

\section{INTRODUCTION}

To produce high-density magneto-optical (MO) disks, stable formation of small marks - which is essential for a high signal-to-noise ratio $(\mathrm{S} / \mathrm{N})$ - must be achieved. We have investigated the relationship between the surface roughness and noise characteristics in MO disks and have reported that using a high-quality flat underlayer is an effective way to decrease the disk noise $\left(\mathrm{N}_{\mathrm{d}}\right)$ of non-recorded disks[1]. However, the flat underlayer causes irregular domain shapes, so that the recording noise $\left(\mathrm{N}_{\mathrm{r}}\right)$ generated by recording marks increases. That is, the flat underlayer prevents the stable formation of domain marks. If this $\mathrm{N}_{\mathrm{r}}$ can be suppressed (i.e., stable formation of small marks is achieved) on the smoothed underlayer needed to bring about low $\mathrm{N}_{\mathrm{d}}$. we can obtain high S/N MO disks. Therefore, methods of decreasing $\mathrm{N}_{\mathrm{r}}$ and the details of the domain formation on the smoothed underlayer need to be studied.

One of the possible ways to decrease $\mathrm{N}_{\mathrm{I}}$ through the stable formation of domain marks is achieving a steep slope of coercivity in the vicinity of the temperature at which the domain shape is fixed, and it is known that the temperature dependence of coercivity is changed by varying the $\mathrm{TbFeCo}$ composition. We began the work reported here by investigating the $\mathrm{N}_{\mathrm{r}}$ for various TbFeCo compositions in MO disks with a smoothed underlayer and compared this to the $\mathrm{N}_{\mathrm{r}}$ in disks with a rough underlayer. We found an interesting $N_{r}$ dependence on the compensation temperature $\left(\mathrm{T}_{\text {comp }}\right.$ ) used for flat underlayer disks. We also examined the domain wall motion, which is important in the domain formation for both flat and rough underlayer disks.

\section{EXPERIMENTAL}

We prepared MO disks with TbFeCo films that had a $\mathrm{T}_{\text {comp }}$ from $-180^{\circ} \mathrm{C}$ to $140^{\circ} \mathrm{C}$ by magnetron sputtering. The disk structure was as follows: glass substrate (pregrooved with a photo-polymerized resin) / nitride underlayer $(85 \mathrm{~nm}) / \mathrm{TbFeCo}(25 \mathrm{~nm}) /$ nitride $(20 \mathrm{~nm}) /$
Al alloy $(50 \mathrm{~nm})$. The surface roughness of the nitride underlayer was controlled by using two different sputtering processes. This resulted in disks with a flat underlayer (F-disks) or a rough underlayer (R-disks). Mean roughness $(\mathrm{Ra})$ of underlayer surfaces in $\mathrm{F}$ - and $\mathrm{R}$ disks, which were measured using an atomic force microscope, were $0.28 \mathrm{~nm}$ and $0.47 \mathrm{~nm}$, respectively.

The dynamic tester used here had an 830-nm laser diode and an objective lens with a numerical aperture of 0.55 . When measuring $\mathrm{S} / \mathrm{N}$, a linear velocity of $4.2 \mathrm{~m} / \mathrm{s}$. a recording frequency of $2.5 \mathrm{MHz}$, and a reading power of $1 \mathrm{~mW}$ were used.

\section{RESULTS AND DISCUSSION}

Figure 1 shows the $T_{\text {comp }}$ dependence of the $N_{r}$ normalized by the signal. Domain marks were recorded by applying the optimal external field for each disk. The $\mathrm{S} / \mathrm{N}$ was obtained by integrating the noise within a frequency range of 0 to $5 \mathrm{MHz}$ to reflect the $\mathrm{N}_{\mathrm{r}}$ level at low frequencies. Among the R-disks, rare-earth (RE) rich disks have a lower recording noise than those rich in transition metals (TM). The reason is thought to be that RE-rich disks have steeper slopes of coercivity than those of TM-rich disks. (This can be seen from Fig. 3 which shows the nucleation field, $\mathrm{H}_{\mathrm{n}}$ rather than coercivity; these two properties have almost the same values.) The F-disks, however, have their lowest $\mathrm{N}_{\mathrm{r}}$ when $-70^{\circ} \mathrm{C} \leqq$ $\mathrm{T}_{\text {comp }} \leqq 10^{\circ} \mathrm{C}$. The recording noise of RE-rich F-disks is remarkably large even though the slope of their coercivity is steeper than that of the other F-disks (see Fig. 4). This shows that the domain shape in the RE-rich F-disks is more irregular than in the other F-disks. Therefore, it appears that the domain shape in the F-disks is strongly influenced by forces other than coercivity.

The coercivity is the field needed to reverse magnetization when there are no domains. However, we believe that where domains exist. studying the magnetic properties can provide an understanding of the domain wall motion which influences the domain shape. Thus, we 
investigated the domain wall motion by examining the threshold field at which the domains begin to expand or contract in both $\mathrm{F}$ - and $\mathrm{R}$-disks.

We measured an expanding field $\left(\mathrm{H}_{\text {exp }}\right)$, a contracting field $\left(\mathrm{H}_{\mathrm{cnt}}\right)$ and a nucleation field $\left(\mathrm{H}_{\mathrm{n}}\right)$ as shown in Fig, 2 with the Kerr effect measurement apparatus and using three domain lengths $(0.62,0.82$ and $1.1 \mu \mathrm{m})$. In this measurement, direct-grooved glass substrates were used because the temperature rose to $200^{\circ} \mathrm{C}$.

The results for the $\mathrm{R}$ - and F-disks are shown in Figs. 3 and 4 , respectively. Also, $\mathrm{H}_{\exp }$ normalized by $\mathrm{H}_{\mathrm{n}}\left(\mathrm{H}_{\mathrm{exp}}{ }^{\prime}\right.$ $\left.\mathrm{H}_{n}\right)$ and $\mathrm{H}_{\mathrm{cnt}}$ normalized by $\mathrm{H}_{\mathrm{n}}\left(\mathrm{H}_{\mathrm{cnt}} / \mathrm{H}_{\mathrm{n}}\right)$ are shown in Figs. 5 and 6 , respectively. A small (or large) $H_{\text {exp }}$ means a large (or small) expanding force inherent in the domain wall. Similarly, a small (or large ) $\mathbf{H}_{\mathrm{cnt}}$ means a large (or small) contracting force of the domain wall. Figure 3 shows that $H_{e x p}, H_{c n t}$, and $H_{n}$ all have similar values and are independent of the TbFeCo composition and the domain size in the R-disks. In the hightemperature region (about $\geqq 100^{\circ} \mathrm{C}$ ) where the domains were formed, $\mathrm{H}_{\mathrm{exp}}$ was smaller than $\mathrm{H}_{\mathrm{cnt}}$ (that is, expansion was easy) in a $\mathrm{TM}$-rich disk, and $\mathrm{H}_{\mathrm{cnt}}$ was smaller than $\mathrm{H}_{\exp }$ (that is, contraction was easy) in a $\mathrm{RE}$ -

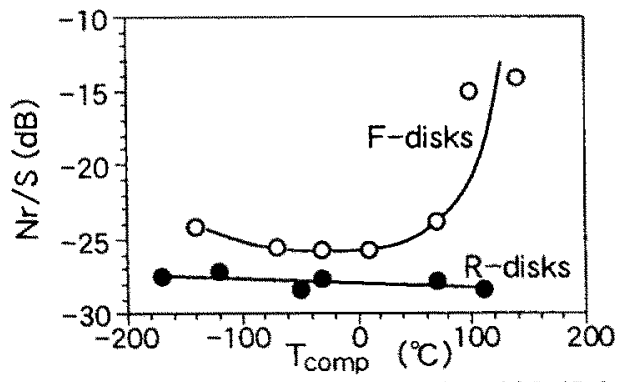

Fig. 1 Recording noise normalized by signal $\mathrm{N}_{\mathrm{r}} / \mathrm{S}$ for various $\mathrm{T}_{\text {comp. }}$. $\mathrm{Nr}_{\mathrm{r}}$ is integrated within a frequency range of 0 to $5 \mathrm{MHz}$. rich disk as shown in Fig. 5. Takahashi et al have reported that during the thermomagnetic domain formation an expanding force acts on a recorded domain wall in TM-rich films and a contracting force acts on the wall in RE-rich films[3]. The results shown in Fig. 5 support Takahashi's findings.

In the F-disks, $\mathrm{H}_{\mathrm{cnt}}$ is always smaller than $\mathrm{H}_{\text {exp }}$, at least within the range of $-170^{\circ} \mathrm{C} \leqq \mathrm{T}_{\text {comp }} \leqq 100^{\circ} \mathrm{C}$ (Fig. 4). The contracting force is so large that the domain begins to contract even when the external field is half of $\mathrm{H}_{n}$. The contracting field is larger with smaller domain sizes and higher $\mathrm{T}_{\text {comp. }}$. This contracting field seems to affect the recording power. For example, the recording power needed to write $0.62-\mu \mathrm{m}$ domains in F-disks is 6.8 to 6.9 $\mathrm{mW}$, compared to $6.5 \mathrm{~mW}$ for R-disks. That is, at the same recording power, smaller domains were formed in the F-disks than in the R-disks. We believe the domain shrinks during the domain formation due to the large contracting force that acts on the domain wall in F-disks.

Next, we used Huth's theory[3] to examine the forces affecting the domain wall in the R-and F-disks. When the domain is formed, the position of the domain wall is determined by the balance between the demagnetization field $\left(\mathrm{H}_{\mathrm{d}}\right)$, the external field $\left(\mathrm{H}_{\mathrm{ext}}\right)$, and other forces as expressed in the following equation[3].

$\left|-\mathrm{H} 1-\mathrm{H} 2+\mathrm{H}_{\mathrm{d}}+\mathrm{H}_{\mathrm{ext}}\right| \leq \mathrm{H}_{\mathrm{c}}$ where

$\mathrm{H} 1=\sigma_{\mathrm{w}} / 2 \mathrm{rM}_{\mathrm{S}}$

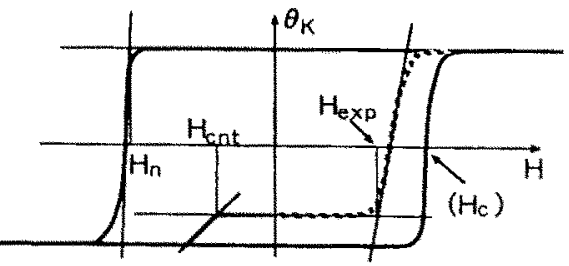

Fig. 2 An example of Kerr hysteresis loops
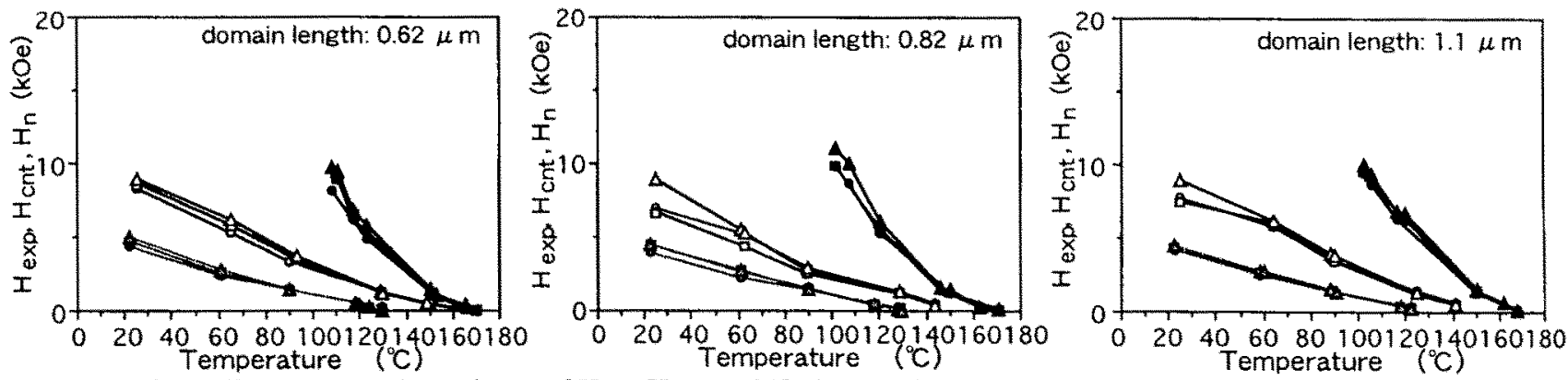

Fig. 3 Temperature dependence of $\mathrm{H}_{\mathrm{cnt}}, \mathrm{H}_{\exp }$ and $\mathrm{H}_{\mathrm{n}}$ in R-disks.

, 용 and $\mathrm{A}$ indicate $\mathrm{H}_{\mathrm{cnt}}, \mathrm{H}_{\mathrm{exp}}$ and $\mathrm{H}_{\mathrm{n}}$ of a TM-rich disk, respectively.

$O, \square$ and $\triangle$ indicate those of a $T_{\text {comp }} \approx-70^{\circ} \mathrm{C}$ disks and, $\mathbf{U}$ and $\mathbf{\Delta}$ indicate those of an $\mathrm{RE}$-rich disk.
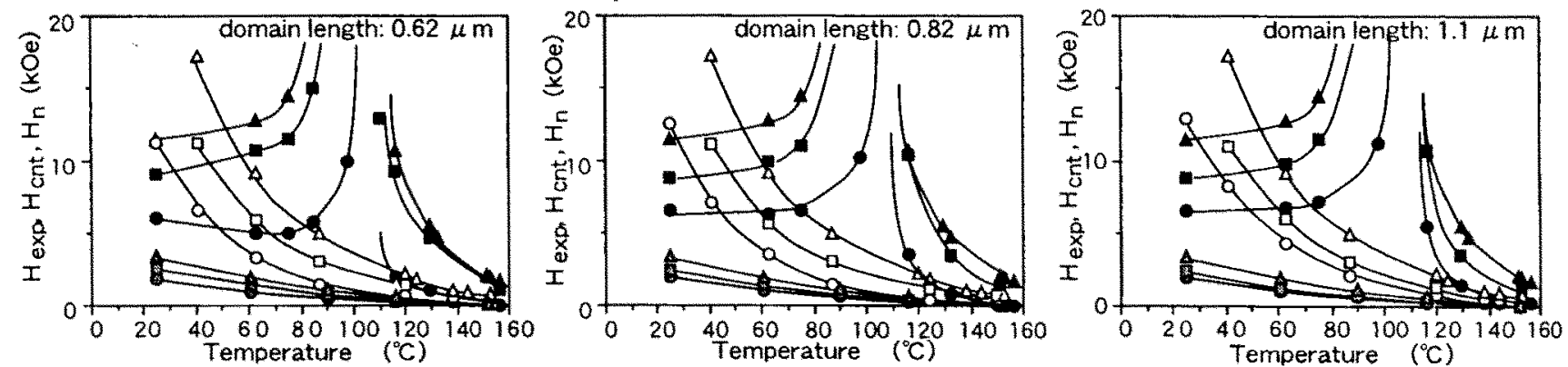

Fig. 4 Temperature dependence of $\mathrm{H}_{\mathrm{cnt}}, \mathrm{H}_{\mathrm{exp}}$ and $\mathrm{H}_{\mathrm{n}}$ in $\mathrm{F}$-disks, using the symbles given in Fig. 3 but with $\mathrm{T}_{\mathrm{comp}} \approx 0^{\circ} \mathrm{C}$. 

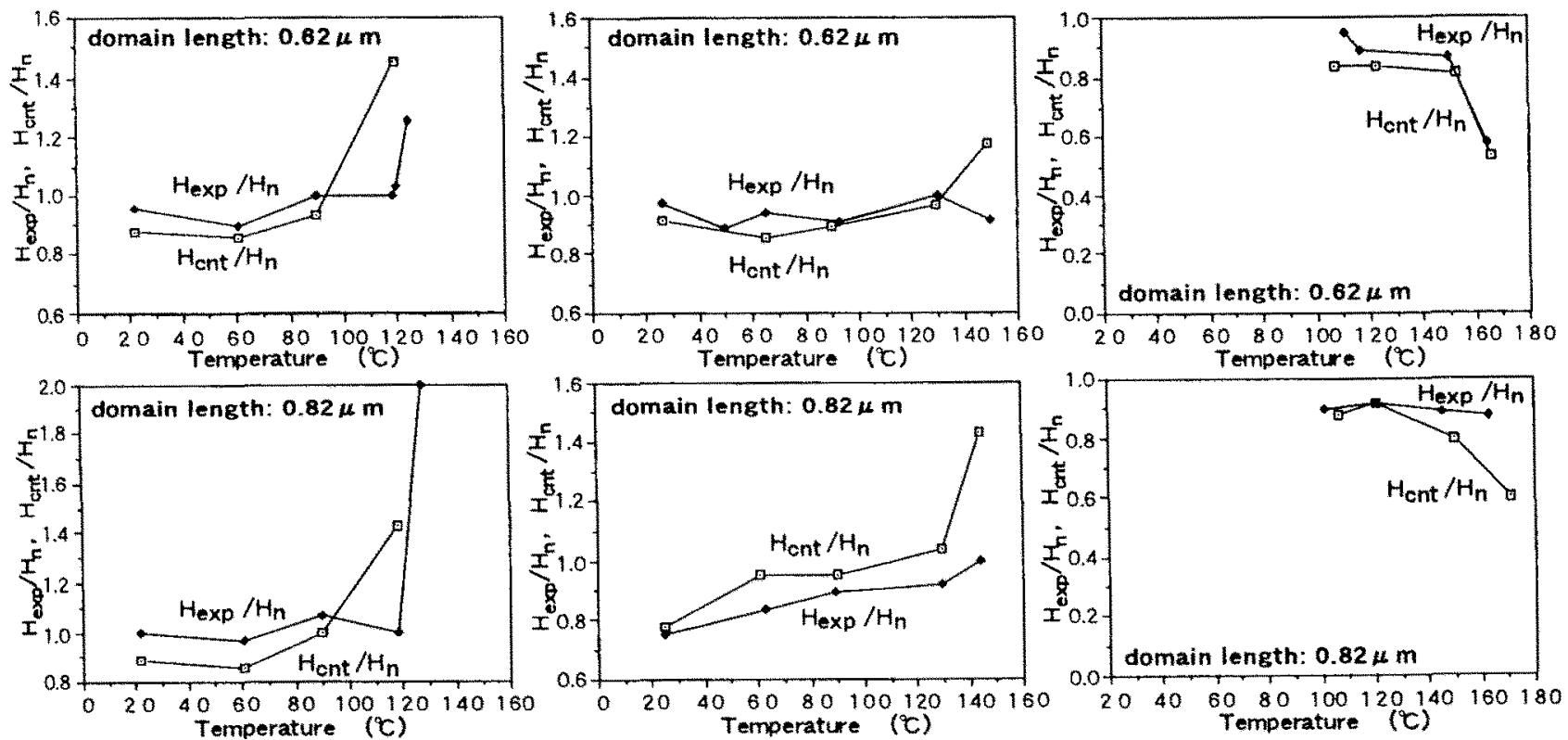

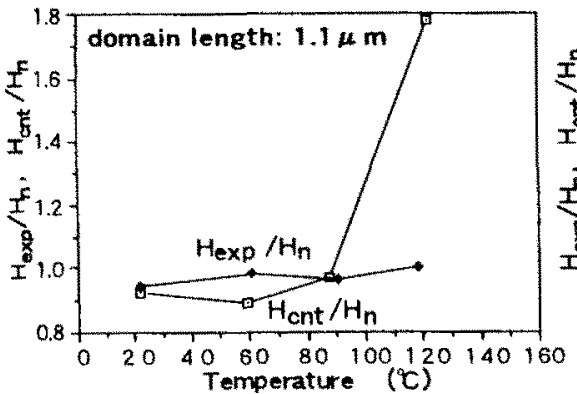

(a) TM-rich disks

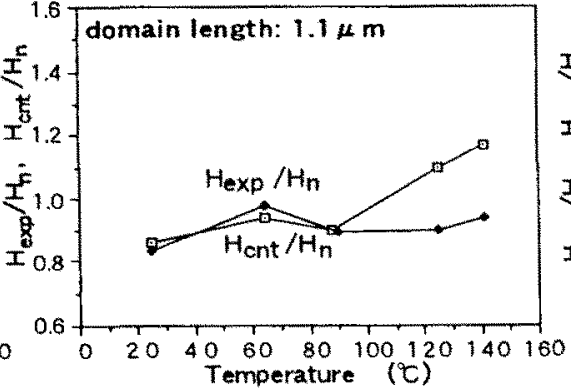

(b) $T_{\text {comp }} \approx-70^{\circ} \mathrm{C}$ disks

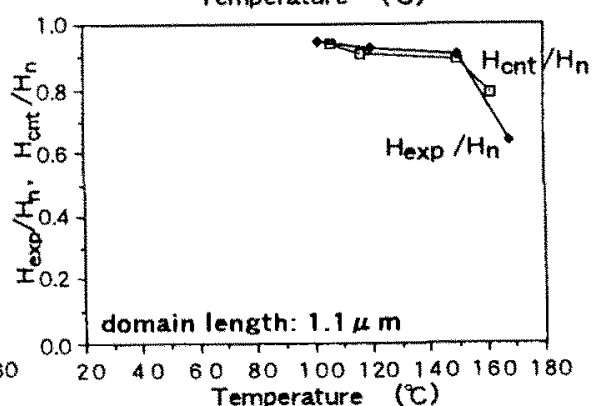

(c) RE-rich disks

Fig. $5 \mathrm{H}_{\mathrm{cnt}} / \mathrm{H}_{\mathrm{n}}, \mathrm{H}_{\mathrm{exp}} / \mathrm{H}_{\mathrm{n}}$ in R-disks.

and

$\mathrm{H}_{2}=\left(1 / 2 \mathrm{M}_{\mathrm{s}}\right) \cdot\left(\partial \sigma_{w} / \partial \mathrm{T}\right) \mathrm{r} \cdot(\partial \mathrm{T} / \partial \mathrm{r})$.

Here, $M_{s}$ is the saturation magnetization. In Eq. (1), the minus sign denotes the direction of contracting domains and the plus sign denotes the direction of expanding domains. In the experimental results shown in Figs. 3 to 6 , H2 equals 0 because the temperature was constant in the recording film during the measurement. This definition of $\mathrm{Hl}$ means the contracting force originates from the curvature of the domain wall.

In F-disks, $\mathrm{H}_{\mathrm{cnt}}$ is always smaller than $\mathrm{H}_{\text {exp. This }}$ result is expressed using Eq. (1),

$-\mathrm{H} 1+\mathrm{H}_{\mathrm{d}}+\mathrm{H}_{\mathrm{exp}}=\mathrm{H}_{\mathrm{c}}$

$-\mathrm{H} 1+\mathrm{H}_{\mathrm{d}}-\mathrm{H}_{\mathrm{cnt}}=-\mathrm{H}_{\mathrm{C}}$

$\mathrm{H}_{\text {exp }}>\mathrm{H}_{\mathrm{cnt}}$.

From Eqs. (4), (5) and (6), the relation between $\mathrm{Hl}$ and $\mathrm{H}_{\mathrm{d}}$ can be expressed as

$\mathrm{Hl}>\mathrm{H}_{\mathrm{d}}$.

Equation (7) means the contracting force is larger than the expanding force in the case of F-disks. The domain appears to begin shrinking at a small applied field $\left(\mathrm{H}_{\mathrm{cnt}}\right)$ because the contracting force acts on the domain wall more strongly than does the expanding force. To expand the domain, a large expanding field $\left(\mathrm{H}_{\text {exp }}\right)$ has to be applied because an extra force that can compensate for the contracting force in the domain wall is needed. In Fig. 6, the difference between $\mathrm{H}_{\text {exp }} / \mathrm{H}_{\mathrm{n}}$ and $\mathrm{H}_{\mathrm{cnt}} / \mathrm{H}_{\mathrm{n}}$ increases as the domains become smaller in all the three TbFeCo compositions. We think this occurs because $\mathrm{Hl}$ (the contracting force) increases as the radius of the domain ( $r$ ) in Eq. (2) decreases. When comparing samples with the same domain radius, we found the difference between $\mathrm{H}_{\text {exp }} / \mathrm{H}_{\mathrm{n}}$ and $\mathrm{H}_{\mathrm{cnt}} / \mathrm{H}_{\mathrm{n}}$ in an RE-rich F-disk to be larger than that in a TM-rich F-disk. We explain this as follows. $\mathrm{M}_{\mathrm{s}}$ decreases in an RE-rich film, and $\mathrm{Hl}$ increases with the decreasing $\mathrm{M}_{\mathrm{s}}$ in Eq. (2); moreover, $\mathrm{H}_{\mathrm{d}}$ (acting as an expanding force ) decreases with the decreasing $M_{5}$. Therefore, the contracting force in the RE-rich disk is larger than that in the TM-rich disk. Thus, in the case of F-disks, we can explain the behavior of $\mathrm{H}_{\mathrm{cnt}}$ and $\mathrm{H}_{\mathrm{exp}}$ by using Huth's equation.

On the contrary, it is hard to explain the behavior of $\mathrm{H}_{\mathrm{cnt}}$ and $\mathrm{H}_{\text {exp }}$ in the R-disks by using Huth's equation. For example, within certain temperature regions (below $90^{\circ} \mathrm{C}$ for the $\mathrm{TM}$-rich disk and $\mathrm{T}_{\text {comp }}=-70^{\circ} \mathrm{C}$ disk, or the entire measuring range for the $\mathrm{RE}$-rich disk), $\mathrm{H}_{\exp }$ and $\mathrm{H}_{\mathrm{cnt}}$ are almost equal and are independent of the $\mathrm{TbFeCo}$ composition and the domain size. When $\mathrm{H}_{\text {exp }}=\mathrm{H}_{\text {cnt }}$, from Eqs. (4) and (5), the relation between the expanding and contracting forces is

$$
\mathrm{H} 1=\mathrm{H}_{\mathrm{d}} \text {. }
$$

Equation (8) means that the term for the domain wall energy and the term for the demagnetization energy remain the same even if the TbFeCo composition (which is related to $\sigma_{w}, M_{s}$ and $H_{d}$ in Eqs. (1) and (2)) and the domain size (related to $\mathrm{r}$ and $\mathrm{H}_{\mathrm{d}}$ in Eqs.(1) and (2)) are 

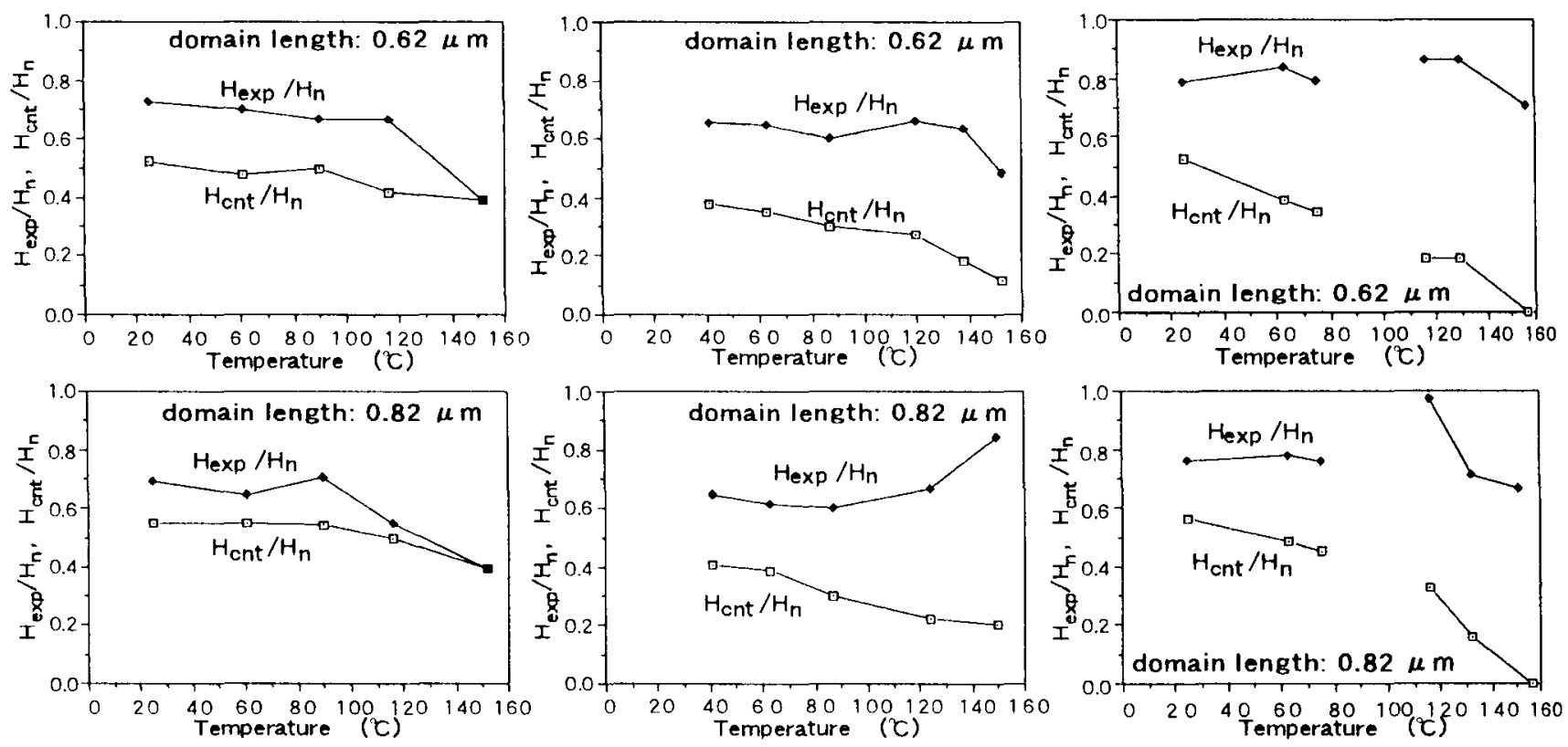

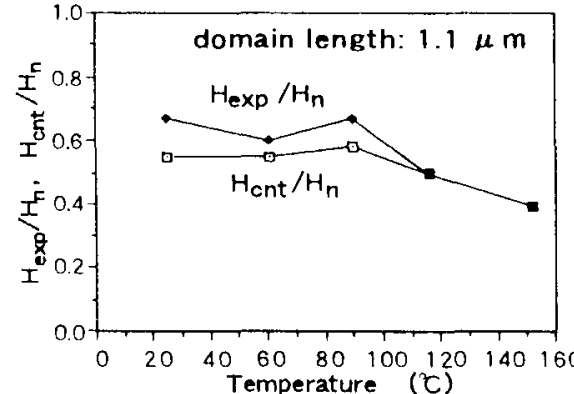

(a) TM-rich disks

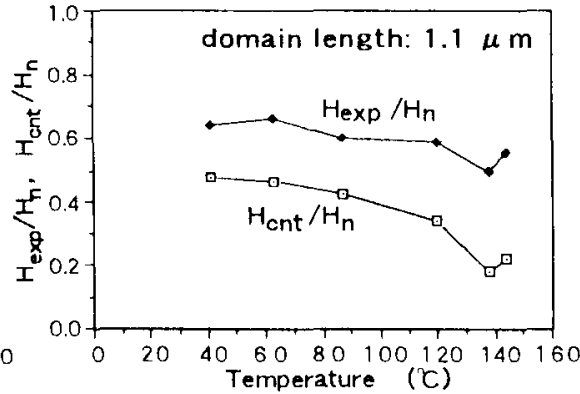

(b) $\mathrm{T}_{\mathrm{comp}} \approx \mathrm{O}^{\circ} \mathrm{C}$ disks

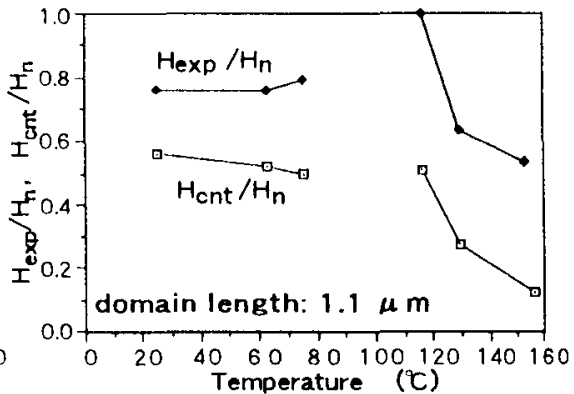

(c) RE-rich disks

Fig. $6 \mathrm{H}_{\mathrm{cnt}} / \mathrm{H}_{\mathrm{n}}, \mathrm{H}_{\mathrm{exp}} / \mathrm{H}_{\mathrm{n}}$ in F-disks.

varied.

Possible causes of the difference in the domain wall motion between the $\mathrm{F}$ - and $\mathrm{R}$-disks include the variance in the magnetic properties of $\sigma_{\mathrm{w}}, \mathrm{K}_{\mathrm{u}}$, and so on, and the variance in the number of domain wall pinning site. It was suggested that the "defects" with large or small magnetic parameters may act as pinning sites by the simulation[4]. We believe that the smoothed underlayer surface reduces the spatial variation of the magnetic parameters, so that Fdisks have fewer pinning sites than the R-disks[5]. The large recording noise in the $\mathrm{RE}$-rich $\mathrm{F}$-disks may come from the strong contracting force combined with few pinning sites, which leads to high wall mobility. The reason why $\mathrm{H}_{\mathrm{exp}}$ and $\mathrm{H}_{\mathrm{cnt}}$ are almost equal may be the action of the large pinning force by many pinning sites in $\mathrm{R}$-disks. Our experimental findings suggest that in a recording film with many pinning sites, we must be cautious in using Huth's equation.

\section{CONCLUSION}

We have investigated the recording noise $N_{r}$ and the domain wall motion for various TbFeCo compositions in $\mathrm{MO}$ disks with a flat underlayer as compared to disks with a rough underlayer. In the case of the R-disks, $N_{r}$ was suppressed as $\mathrm{T}_{\text {comp }}$ increased, but the $\mathrm{F}$-disks had their lowest $\mathrm{N}_{\mathrm{r}}$ when $-70^{\circ} \mathrm{C} \leqq \mathrm{T}_{\text {comp }} \leqq 10^{\circ} \mathrm{C}$. In RE-rich F-disks, $N_{r}$ was very large even though the slope of coercivity was steep; this may indicate the presence of a strong contracting force.

In F-disks, we found that the contracting force increased with smaller domain sizes and with increasing

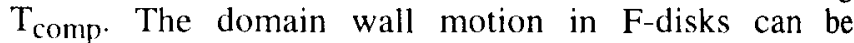
explained by using Huth's theory. However, in R-disks, the domain wall motion doesn't depend on the $\mathrm{TbFeC} 0$ composition or the domain size due to many domain wall pinning sites in these disks.

\section{REFERENCES}

[1] J. Ushiyama, H. Awano, M. Miyamoto, K. Andoh and M. Takahashi, Technical Digest of ISOM'95, Fr-A4 (1995), p 133.

[2] M. Takahashi, S. N. Gadetsky and M. Mansuripur, Proceedings of MORIS'94, J. Magn. Soc. Jpn., 19, Supplement No. S1(1995), p395.

[3] B. G. Huth, IBM J. Res. Dev., 18 (1974) p100.

[4] R. Giles and M. Mansuripur, Comput. Phys., MAR/APR, (1991)p204.

[5] T. Kohashi, H. Matsuyama, C. Haginoya, K. Koike, H. Miyamoto, J. Ushiyama and H. Awano, Proc. of MORIS'96, J. Magn. Soc. Jpn., 20, Supplement No. S1 (1996), p303. 